\title{
Radial Extracorporeal Shockwave Therapy and A Supervised Exercise Programme in Patients with Lateral Elbow Tendinopathy? A Research Protocol
}

\author{
Dimitrios Stasinopoulos ${ }^{1 *}$, Daphne Bakalidou², Magdalini Stamou ${ }^{3}$ \\ ${ }^{1}$ Assistant Professor, Physiotherapy, Dept. of Physiotherapy, Faculty of Health and Caring Sciences, University of West Attica, \\ Member of Laboratory of Neuromuscular \& Cardiovascular Study of Motion (LANECASM)
}

${ }^{2}$ Associate Professor, Physiotherapy, Dept. of Physiotherapy, Faculty of Health and Caring Sciences, University of West Attica, Member of Laboratory of Neuromuscular \& Cardiovascular Study of Motion (LANECASM)

${ }^{3}$ EDIP, Physiotherapy, Dept. of Physiotherapy, Faculty of Health and Caring Sciences, University of West Attica, Member of Laboratory of Neuromuscular \& Cardiovascular Study of Motion (LANECASM)

*Corresponding author: Dimitrios Stasinopoulos, Department of Physiotherapy, Assistant Professor, Physiotherapy, Faculty of Health and Caring Sciences, University of West Attica, Member of Laboratory of Neuromuscular \& Cardiovascular Study of Motion (LANECASM), Agiou Spyridonos 28, Egaleo 12243, Athens -Greece

\section{ARTICLE INFO}

Received: 慧 October 19, 2021

Published: 幽 October 26, 2021

Citation: Dimitrios Stasinopoulos, Daphne Bakalidou and Magdalini Stamou. Radial Extracorporeal Shockwave Therapy and A Supervised Exercise Programme in Patients with Lateral Elbow Tendinopathy? A Research Protocol. Biomed J Sci \& Tech Res 39(4)-2021. BJSTR. MS.ID.006329.

\begin{abstract}
Background: One of the two most common tendinopathies of the upper limb is Lateral elbow Tendinopathy (LET). An exercise programme consisting of static stretching exercises of wrist extensors, isometric of wrist extensors, concentric - eccentric training of wrist extensors, Tendon Neuroplastic Training (TNT) of wrist extensors, and strengthening of rotator cuff, scapula muscles exercises and supinator has been recommended for the management of LET. Radial Extracorporeal shockwave therapy (RESWT) is usually used as a supplement to exercise programme. The purpose of the present article will be to make a comparison of the effects of an exercise programme consisting of TNT of wrist extensors, static stretching exercises of wrist extensors, isometric of wrist extensors, concentric - eccentric training of wrist extensors and strengthening of supinator, rotator cuff and scapula muscles exercises and RESWT in the painful point and an exercise programme consisting of TNT of wrist extensors, static stretching exercises of wrist extensors, isometric of wrist extensors, concentric - eccentric training of wrist extensors and strengthening of supinator, rotator cuff and scapula muscles exercises and RESWT, scanning and painful (sensitive) point for the treatment of LET.
\end{abstract}

Methods/Design: LET patients will participate in this randomized clinical trial (RCT). Patients will be allocated to two groups randomly. Group A will be treated with TNT of wrist extensors, static stretching exercises of wrist extensors, isometric of wrist extensors, concentric - eccentric training of wrist extensors and strengthening of supinator, rotator cuff and scapula muscles exercises and RESWT in the painful point and group B will be treated with TNT of wrist extensors, static stretching exercises of wrist extensors, isometric of wrist extensors, concentric - eccentric training of wrist extensors and strengthening of supinator, rotator cuff and scapula muscles exercises and RESWT, scanning the relative area and painful (sensitive) point. All patients will receive 20 treatments totally of exercise programme ( 5 treatments / week for 4 weeks). RESWT will be administered without anaesthesia in 3 treatment sessions held at weekly intervals (2000 shocks for painful site and 4000 shocks for scanning the relative area and painful (sensitive) point; $8 \mathrm{~Hz}$; 2,5 bars). Pain (visual analogue scale; Patient-Rated Tennis Elbow Evaluation), function (visual analogue scale; Patient-Rated Tennis Elbow 
Evaluation) and pain-free grip strength will be evaluated at the end of treatment, at 3 months follow-up and at 6 months follow up. The independent t test will be used to determine the differences between groups. A paired t test will be used to determine the difference within groups. The level for statistical significance will be $5 \%$ level of probability. SPSS 21.00 will be used for the statistical analysis.

Discussion: The present RCT will be evaluate the effectiveness of two different applications of RESWT as a supplement to exercise programme in patients with LET.

Trial Registration: The Ethics Committee of the University of West Attica will approve the study.

\section{Background}

Lateral elbow tendinopathy (LET), one of the most common lesions of the arm work-related or sport-related pain disorder, is the most appropriate term to use in clinical practice because all the other terms such as lateral epicondylalgia, lateral epicondylosis, lateral epicondylitis and/or tennis elbow make reference to inappropriate pathophysiological, aetiological, and anatomical terms [1]. LET is a syndrome of pain in the area of the lateral epicondyle [2] that may be failed healing tendon response or degenerative rather than inflammatory [3]. Hence, vascular hyperplasia, proteoglycans and glycosaminoglycans, the increased presence of fibroblasts together with disorganized and immature collagen may all take place in the absence of inflammatory cells [4]. The origin of the extensor carpi radialis brevis (ECRB) is the most commonly affected structure is [4]. The dominant arm is commonly affected, between 30 and 60 years of age is the peak prevalence of LET [2,5] and the disorder appears to be severed and of longer duration in women $[3,6]$. The main complaints of patients with LET are decreased function and pain $[2,3]$. A therapist should be able to reproduce the symptoms by:

a) Resisted wrist extension and/or resisted middle-finger extension with the elbow in extension,

b) Getting the patient to grip an object and

c) Digital palpation on the facet of the lateral epicondyle $[2,3,5]$.

No ideal treatment has emerged for LET management. A conservative approach is advocated by many clinicians as the treatment of choice for LET $[2,3,7,8]$. Physiotherapy is usually recommended for LET patients [2-9]. A wide array of physiotherapy treatments has been recommended for the management of LET [10-14]. Such a variety of treatment techniques suggests that the optimal treatment management is not known, and more research is needed to find out the most effective treatment approach in patients with LET [10-14]. An exercise programme is the most common physiotherapy treatment for LET [2-14]. There are two types of exercise programs: home exercise programs and exercise programs carried out in a clinical setting. A home exercise program is commonly advocated for patients with LET because it can be performed any time during the day without requiring supervision by a physiotherapist. Our clinical experience, however, has shown that patients fail to comply with the regimen of home exercise programs [15]. This problem can be solved by exercise programs performed in a clinical setting under the supervision of a physiotherapist. For the purposes of this report, "supervised exercise program" will refer to such programs. Therefore, such a supervised exercise program will be used in the present trial.

Although a supervised exercise program is an effective treatment approach, a supplement to the exercise program should be found to reduce the treatment period. One such modality is Extracorporeal shockwave therapy (ESWT). ESWT is a safe noninvasive procedure [16] in which a device delivers acoustic energy (shockwaves) through the skin surface onto the affected area. Focused shockwaves are typically generated by electromagnetic or piezoelectric techniques [17]. Radial shockwave (RESWT) is nonfocused and generated by a ballistic source [18]. Recently research has started to be published on RESWT, which is relatively new and a promising modality to treat LET. ESWT may promote the release of angiogenetic growth and proliferating factors (e.g. vascular endothelial growth factor and endothelial nitric oxide synthase) that induces neovascularization for tissue regeneration [17]. Furthermore, ESWT may down-regulate inflammatory mediators and directly suppress nociceptors by hyperstimulation analgesia [19].

RESWT is applied only in the area of pain, in the case of LET in the ECRB. I wonder if it can be applied in a different way, like scanning the whole area for example muscles of the forearm or parallel to the elbow joint line. Perhaps, the combination of the above two ways of application, scanning and painful (sensitive) point, leads to a better clinical result. To our knowledge, the effectiveness of the above combination of RESWT in the management of LET has not been investigated. It is possible to combine a supervised exercise programme with RESWT, scanning and painful (sensitive) point, to see if the combination of the above reported therapeutic approaches offers superior results to supervised exercise programme and RESWT in the painful point in LET patients. Therefore, the aim 
of the present article will be to make a comparison of the effects of an exercise programme consisting of Tendon Neuroplastic Training (TNT) of wrist extensors, static stretching exercises of wrist extensors, isometric of wrist extensors, concentric - eccentric training of wrist extensors and strengthening of supinator, rotator cuff and scapula muscles exercises with RESWT, scanning and painful (sensitive) point and an exercise programme consisting of consisting of Tendon Neuroplastic Training (TNT) of wrist extensors, static stretching exercises of wrist extensors, isometric of wrist extensors, concentric - eccentric training of wrist extensors and strengthening of supinator, rotator cuff and scapula muscles exercises and RESWT in the painful point only for the treatment of LET.

\section{Methods}

To assess the effectiveness of an exercise programme with RESWT in the management of LET, a randomized controlled, monocentre trial will be conducted in the in the Laboratory of Neuromuscular and Cardiovascular Study of Motion (LANECASM) for 24 months. Crossover designs are limited in situations where patients are cured by the intervention and do not have the opportunity to receive the other treatments after crossover, therefore a parallel group design will be used [20]. Three investigators will participate in the study: (1) a physiotherapist (DB) who will perform all baseline and follow-up assessments, and will gain informed consent, (2) a physiotherapist, (MS), who will administer the treatments. and (3) the primary investigator, (DS) who will evaluate the patients to confirm the LET diagnosis and will allocate patients to groups. DB will interview each patient to ascertain clinical characteristics, including patient name, sex, age, duration of symptoms, previous treatment, occupation, affected arm and dominant arm and baseline demographic. To demonstrate statistical clinical significance for all outcome measures on LET a sample size of 25 subjects per group is sufficient. Measuring pain relief and functional outcomes in response to physiotherapeutic interventions such as low-power laser light, clinical effects of $20 \%$ had been reported as clinically meaningful in placebo-controlled studies. Baseline variance for pain and functional outcomes will be set at $25 \%$ in this study. Power calculations will recommend that 25 patients per group is sufficient to detect a $20 \%$ change in outcome measures, assuming that variance will be equivalent to $25 \%$ with a $5 \%$ significant level and $80 \%$ of power. The formula that will be used to determine the proper sample size will be:

$$
N=16 \sigma^{2} / d^{2}
$$

where $d^{2}$ is the effect size and $\sigma^{2}$ is the variability of the data. For example, in our trial $\sigma=25$ and $d=20$. Therefore, the above formula is $\mathrm{N}=16(252) /(202)=16 \times 625 / 400=25$

Patients over 18 years old with lateral elbow pain will been evaluated and examined in the LANECASM in Athens between October 2021 and April 2023. In addition, persons with paraplegia, wheeled chair users and athletes with paraplegia as well as, can participate in the study. All patients will live in Greece, will speak Greek and will be either referred by their physician or physiotherapist or self-referred. Patients will participate in the study if, at the time of presentation, they will have been clinically diagnosed LET for at least 4 weeks. Patients will participate in the trial if they report (a) less pain during resistance supination with the elbow in 90。 of flexion rather than in full extension and (b) pain on the facet of the lateral epicondyle when palpated and (c) pain in at least two of the following four tests [7]:

a) Handgrip dynamometer test.

b) Mill's test (full passive flexion of the wrist)

c) Tomsen test (resisted wrist extension)

d) Resisted middle finger test

Patients will not participate in the study if they have one or more of the following conditions:

a. Radial nerve entrapment

b. Dysfunction in the shoulder, neck (radiculopathy) and/or thoracic region

c. Neurological deficit

d. Had received any conservative treatment for the management of LET in the 4 weeks before entering the study

e. The affected elbow had been operated on

f. Limitations in arm functions; and

g. Local or generalized arthritis [21-24].

A written explanation of the trial prior to entry into the study will be received by all patients. All patients will sign an informed consent to take part in the study. The study will be approved by the Ethics Committee of the University of West Attica. The allocation of the patients to two groups will be done randomly by drawing lots. Patients in Group A will be treated with a supervised exercise programme and RESWT in the painful point and patients in Group $B$ will be treated with the supervised exercise programme and RESWT, scanning the relative area and painful (sensitive) point. All patients will be instructed to use their arm during the course of the study but to avoid activities that will irritate the elbow such as knitting, lifting, driving a car, using a screwdriver, grasping and handwriting. They will also be informed to refrain from taking pain killer drugs or other conservative treatment throughout the course of the study. A treatment diary will be used to monitor Patient compliance. 
Interaction (verbal and non-verbal) and communication between the patient and therapist will be kept to a minimum, and behaviours sometimes used by physiotherapists to facilitate positive treatment outcomes will be purposefully avoided. For example, patients will be given no feedback on their performance in the pre-application and post-application measurements indication or any of the potentially beneficial effects of the treatments [25]. In both groups the elbow will be on the bed extended, the forearm pronated, the wrist in extension (and the hand hanging at the edge of the table. From this position, subjects will flex their wrists and then return to the extension (starting position). In the starting position, subjects will carry out an isometric contraction of wrist extensors. When the isometric contraction finishes the subjects will carry out the eccentric - concentric contraction and so on. The exercise will involve isolated wrist extension and flexion paced to an external audio/visual cue on the patients' smartphone (PR Metronome; http://eumlab.com/pro-metronome/). Subjects will be to track the movement and listen to the sound of the metronome with their eyes. Each beat will be ten seconds apart, so that the pace of the metronome will be setted to 6 beats per minute. This will allow a ten second eccentric, concentric and isometric phase [26]. Both groups will perform three sets of 15 repetitions of slow progressive exercises of the wrist extensors at each treatment, with 1-min rest interval between each set. Subjects will be informed to continue with the exercise even if they complain of mild pain. However, subjects will be informed to stop the exercise if the pain becomes disabling. The disabling and mild pain will be monitored asking the subjects to rate the pain on VAS before and after treatment period. The definition of mild pain will be below 4 on VAS whereas the definition of disabling pain will be above 8 on VAS $[21,23]$. When subjects are able to carry out the exercise programme without experiencing any discomfort or pain, free weights will be used to increase the load.

Both treatment groups will perform static stretching exercises of the wrist extensors. Three times before and three times after the exercises six times totally will be repeated the static stretching exercises at each treatment session, with a 30 second rest interval between each repetition. The other hand will help to be performed the static stretching exercises of the wrist extensors. The patient's elbow will be placed in extended position, the forearm in pronated position, and the wrist in ulnar deviation and flexion according to the patient's tolerance. $30-45$ seconds will be holding this position each time and then releases [21-24]. Furthermore, the scapular and rotator cuff muscles will be strengthened. The strengthening exercises will be included

(i) Shoulder lateral rotation and medial with the elbow in 900 and 00 of abduction

(ii) (Shoulder abduction to 900 with flexed elbow:

\section{(iii) Scaption and}

(iv) Diagonal pattern from flexion to extension [27].

Upper trapezius, rhomboids, serratus anterior and levator scapulae will be also strengthened [28]. Each exercise will be carried out twice at each treatment with 12 repetitions in each set and 1 min rest interval between each set [29]. Subjects will be informed to continue with the exercise even if they will complain of mild pain. However, subjects will be informed to stop the exercise if the pain became disabling. When subjects will be able to carry out the strengthening exercises without experiencing any discomfort or pain, the load will be increased using therabands or free weights.

Finally, the supinator will be strengthened. Strengthening exercises of the supinator will be carried out with the elbow extended on the table, the forearm pronated, the wrist in mid position and the hand hanging over the edge of the table. From this position, the patient will supinate their arm slowly while counting to 15 using chronometer, then return to the starting position (pronation) [30]. The above reported exercise programme will be followed five times per week for 4 weeks and will be individualized on the basis of the patient's description of pain experienced during the process. RESWT will be administered without anaesthesia in the most sensitive point on patient's lateral epicondyle in Group A. Each patient will receive a total of 2000 shocks per session (8 $\mathrm{Hz} ; 2,5$ bars). Each subject will participate in 3 treatment sessions held at weekly intervals. RESWT will be also administered without anaesthesia in Group B. First 2000 shocks ( 8 Hz, 2.5 bars) will be applied to the most sensitive point of the lateral epicondyle and then 2000 shocks to the dorsal part of the forearm using the same frequency and pressure variables. Each patient will participate in 3 treatment sessions held at weekly intervals. Function, drop-out rate and pain will be measured in the present trial. Each subject will be evaluated at the beginning of the treatment (week 0), at the end of treatment (week 4), at 3 months after the end of the treatment (week 16) and at 6 months (28weeks) after the end of treatment.

A visual analogue scale (VAS) will be used to measure pain. 0 $(\mathrm{cm})$ on VAS means "least pain imaginable" and $10(\mathrm{~cm})$ on VAS means "worst pain imaginable". The pain VAS will measure the patient's worst level of pain over the previous $24 \mathrm{~h}$ before each evaluation, and this approach has been shown to be sensitive and valid of the VAS [31]. A visual analogue scale (VAS) will be used to measure function. $0(\mathrm{~cm})$ on VAS means "no function" and 10 $(\mathrm{cm})$ on VAS means "full function". Subjects will be informed to report their overall level of elbow function over the previous $24 \mathrm{~h}$ before each measurement, and this approach has been shown to be sensitive and valid of the VAS [31]. The Patient-Rated Tennis Elbow Evaluation (PRTEE) Questionnaire will be used to assess pain and function. Patients rate their pain and functional limitation on a scale of $0 \mathrm{e} 10$, with 0 being no difficulty and 10 being unable 
to perform. The scores for the various items are used to calculate an overall scale score ranging from 0 (best score) to 100 (worst score). The scale is scored such that $50 \%$ of the score is obtained by summing the five pain items and the remaining $50 \%$ is by obtained by summing the 10 functional (specific and usual activity) items and then divided that subtotal by 2 . This creates a score out of 100 points where 100 is the highest level of pain and disability. The PRTEE questionnaire, provides a very quick (it takes less than 5 min to complete), easy, and standardized quantitative description of pain and functional disability in patients with LET. The Greek version of PRTEE Questionnaire is a reliable and valid measure when administered to patients with LET [32].

Moreover, pain-free grip strength will be used to measure function. The definition of Pain-free grip strength is the amount of force each patient is able to generate with an isometric gripping action before monitoring pain [25]. A Jamar hand dynamometer that had adjustable handles to accommodate different hand sizes will be used to measure force in pounds. The elbow will be placed in extension, forearm in pronation and internal rotation such that the palmar aspect of the hand faced posteriorly with the arm placed by the patient's side. Subjects will be then informed to squeeze the dynamometer handles until they will first experience pain and then to release their grip [25]. The attained grip force will be subsequently recorded. The reading will not be visible to the subjects. Three measures of pain-free grip strength will be determined with a 30 seconds rest interval between each effort, and the mean value of these repetitions will be calculated. The rate of drop-out will also be used as an indicator of treatment outcome. Reasons for patient drop out will be categorised as follows:

a) Not returned for follow-up

b) Request for an alternative treatment and

c) A withdraw without reason.

The change from the beginning of the treatment will be calculated for each follow-up. The independent $t$ test will be used to determine the differences between groups. A paired $t$ test will be used to analyze the difference within groups between beginning of the treatment and end of treatment. The level for statistical significance will be adopted as a $5 \%$ level of probability. The statistical analysis will be carried out using the SPSS 21.00 statistical software.

\section{Discussion}

The main aim of this RCT is to investigate the effectiveness of two physiotherapy treatments in improving function, strength, and pain in LET patients at the end of treatment, at 3 months follow-up and at 6 months follow up. It is expected to examine the following null hypothesis: "there is no difference in function and pain for subjects undergoing physiotherapy intervention with RESWT in the painful point and RESWT, scanning the relative area and painful (sensitive) point". Many treatments have been recommended for the management of LET. However, there is not the gold standard treatment. Eccentric contraction is recommended [33,34] over other types of contractions for the management of LET. On the other hand, Malliaras and his colleagues [35] proposed that therapists should consider eccentric-concentric loading alongside or instead of eccentric loading. Martinez-Silvestrini et al. [36] concluded that, unlike Achilles' tendinopathy, LET is often related to forceful grip activities requiring isometric contraction, which would be more beneficial than eccentric contraction in LET. Recently, isometric exercises have been recommended to manage and reduce tendon pain improving the strength at the angle of contraction without producing inflammatory signs [37]. The exercise program in LET should include strengthening exercises for supinator, rotator cuff and scapular muscles [38,39]. Moreover, proprioception is also reduced in LET patients [40]. Techniques to improve the reduced proprioception is also recommended. Furthermore, tendon neuroplastic training (TNT) is needed combining isometric or isotonic strength training with an externally paced audio or visual cue [41]. Finally, stretching has positive effects in the management of tendon injuries such as LET. The aim of stretching is to orientate the new collagen fibres, experience consequently less strain during joint motion and lengthen the muscle-tendon unit, [42-44].

Although a supervised exercise program is an effective treatment approach, a supplement to the exercise program should be found to reduce the treatment period. One such modality is RESWT which is a relatively new treatment approach, but it is reported to be used by clinicians worldwide [45]. Karanasios et al. [46] found in their systematic review that ESWT (radial and focused) offers no benefit in the management of LET patients. On the other hand, two published meta-analyses in 2020 [47,48] reported superior outcomes of the ESWT (radial and focused) compared with sham or other passive treatments in pain reduction and function improvement. The above recommendations should be treated with caution because the three reported mentioned studies examined the effectiveness of ESWT as monotherapy in the management of LET. However, ESWT is rarely applied as a monotherapy in clinical practice. ESWT does not use as a substitute for exercise but as a supplement to exercise program. The present study will be the first trial to examine the effectiveness of RESWT, scanning the relative area and painful (sensitive) point, on chronic LET. A radial shock wave stimulates a much larger area of tissue than a focused shock wave. The effective focal zone of the latter is very small [49], thus, the area of affected tissue that can be treated is also small. The radial shock wave allows treating the original site of the "problem" (e.g. the lateral epicondyle), as well as other affected areas. 
One previous pilot study assessed the effectiveness of this RESWT approach on chronic LET [50]. However, the purpose of this article was to evaluate and compare the efficacy of radial and focused shock wave therapies applied to treat LET and no to compare two applications of RESWT in the management of LET, as in our study. The findings of these two trials will encourage the design of future well-designed trials that might produce strong evidence for the effectiveness of RESWT on LET management. RESWT is a dose-response modality and the optimal treatment dose has obviously not yet been discovered. Future studies are needed to standardize RESWR parameters in the management of LET (acute, chronic and calcific).

\section{Conclusion}

This is the first trial to examine the efficacy of RESWT, scanning the relative area and painful (sensitive) point, in the treatment of LET. It is expected, the conclusion of the trial to improve the scientific knowledge providing evidence that using RESWT is an effective and safe tool in the treatment of LET symptoms, specifically function and pain.

\section{References}

1. Stasinopoulos D, Johnson MI (2006) Lateral elbow tendinopathy is the most appropriate diagnostic term for the condition commonly referred to as lateral epicondylitis. Medical Hypotheses 67: 1399-1401.

2. Bisset LM, Vicenzino B (2015) Physiotherapy management of lateral epicondylalgia. J Physiotherapy 61: 174-181.

3. Coombes BK, Bisset L, Vicenzino B (2015) Management of Lateral Elbow Tendinopathy-One Size Does Not Fit All. J Orthop Sports Phys Ther 17 $1-38$.

4. Kraushaar B, Nirschl R (1999) Current concepts review - tendinosis of the elbow (tennis elbow). Clinical features and findings of histological immunohistochemical and electron microscopy studies. J Bone Joint Surg Am 81: 259-285.

5. Vicenzino B, Wright A (1996) Lateral epicondylalgia I: epidemiology, pathophysiology, aetiology and natural history. Phys Ther Rev 1: 23-34.

6. Waugh E, Jaglal S, Davis A, Tomlinson G, Verrier M (2004) Factors associated with prognosis of lateral epicondylitis after 8 weeks of physical therapy. Arch Phys Med Rehabil 85: 308-318.

7. Haker E (1993) Lateral epicondylalgia: diagnosis, treatment and evaluation. Crit Rev Phys Rehabil Med 5: 129-154.

8. Hong QN, Durand MJ, Loisel P (2004) Treatment of lateral epicondylitis where is the evidence? Joint Bone Spine 71: 369-373.

9. Stasinopoulos D, Johnson MI (2004) Physiotherapy and tennis elbow/ lateral epicondylitis. Letter. Rapid response to Assendelft et al. (2003) article Tennis elbow: BMJ.

10. Trudel D, Duley J, Zastrow I, Kerr E, Davidson R, MacDermid J (2004) Rehabilitations for patients with lateral epicondylitis: a systematic review. J Hand Ther 17: 243-266.

11. Smidt N, Assendelft W, Arola H (2003) Effectiveness of physiotherapy for lateral epicondylitis: a systematic review. Ann Med 35: 51-62.

12. Labelle H, Guibert R, Joncas J, Newman N, Fallaha M, et al. (1992) Lack of scientific evidence for the treatment of lateral epicondylitis of the elbow: an attempted meta-analysis. J Bone Joint Surg 74: 646 - 651.
13. Wright A, Vicenzino B (1997) Lateral epicondylalgia II: therapeutic management. Phys Ther Rev 2: 39-48.

14. Bisset L, Paungmali A, Vicenzino B (2005) A systematic review and meta-analysis of clinical trials on physical interventions for lateral epicondylalgia. British Journal of Sports Medicine 39: 411-422.

15. Stasinopoulos, D, Johnson MI (2004) Treatment- management for tendinopathy. Rapid response to Khan et al. (2002) article Time to abandon the 'tendinitis' myth. BMJ.

16. Ioppolo F, Rompe JD, Furia JP, Cacchio A (2014) Clinical application of shock wave therapy (SWT) in musculoskeletal disorders. Eur J PhysRehabil Med 50: 217-230.

17. Wang CJ (2012) Extracorporeal shockwave therapy in musculoskeletal disorders. J OrthopSurg Res 7: 11.

18. Speed C (2014) A systematic review of shockwave therapies in soft tissue conditions: Focusing on the evidence. Br J Sports Med 48: 15381542.

19. Notarnicola A, Moretti B (2012) The biological effects of extracorporeal shock wave therapy (eswt) on tendon tissue. Muscles Ligaments Tendons J 2: 33-37.

20. Johannsen F, Gam A, Hauschild B, Mathiesen B, Jensen L. Rebox: (1993) an adjunct in physical medicine? Arch Phys Med Rehabil 74: 438-440.

21. Manias P, Stasinopoulos D (2006) A controlled clinical pilot trial to study the effectiveness of ice as a supplement to the exercise programme for the management of lateral elbow tendinopathy. British Journal of Sports Medicine 40: 81-85.

22. Pienimaki T, Tarvainen T, Siira P, Vanharanta H (1996) Progressive strengthening and stretching exercises and ultrasound for chronic lateral epicondylitis. Physiotherapy 82: 522-530.

23. Stasinopoulos D, Stasinopoulos I (2006) Comparison of effects of Cyriax physiotherapy, a supervised exercise programme and polarized polychromatic non-coherent light (Bioptron light) for the treatment of lateral epicondylitis. Clinical Rehabilitation 20: 12-23.

24. Stasinopoulos D, Stasinopoulos I, Manias P, Stasinopoulou K (2010) Comparison of effects of a home exercise programme and a supervised exercise programme for the management of lateral elbow tendinopathy, British Journal of Sports Medicine 44: 579-583.

25. Vicenzino B, Wright A, Collins C (1996) The initials effects of a cervical spine manipulative physiotherapy treatment on the pain and dysfunction of lateral epicondylalgia. Pain 68: 69-74.

26. Welsh P (2018) Tendon neuroplastic training for lateral elbow tendinopathy: 2 case reports. J Can Chiropr Assoc 62(2): 98-104.

27. Bhatt JB, Glaser R, Chavez A, Yung E (2013) Middle and lower trapezius strengthening for the management of lateral epicondylalgia: a case report. J Orthop Sports Phys Ther 43(11): 841-7.15.

28. Sharma M, Eapen C, Kamath J (2015) Effect of adding rotator cuff strengthening to therapeutic ultrasound and wrist extensor eccentric exercise for lateral epicondylalgia - a randomized clinical trial. Int J Health Sci Res 5(7): 250-257.

29. Stasinopoulos D, Stasinopoulos I, Stasinopoulou K (2014) A pilot trial to study the effectiveness of an exercise programme in the treatment of rotator cuff tendinopathy. Journal of Biology of Exercise 10(2): 69-77.

30. Demosthenous M, Stasinopoulos D, Lamnisos D (2017) Comparison the effectiveness of eccentric - concentric training of wrist extensors and eccentric - concentric training combined with supinator strengthening in healthy population J Orthop Res Physiother 3: 036.

31. Stratford P, Levy D, Gauldie S, Levy K, Miseferi D (1987) Extensor carpi radialis tendonitis: a validation of selected outcome measures. Physiother Can 39: 250-255. 
32. Stasinopoulos D, Papadopoulos C, Antoniadou M, Nardi L (2015) Greek adaptation and validation of the Patient-Rated Tennis Elbow Evaluation (PRTEE). J Hand Ther 28(3): 286-290.

33. Raman J, MacDermid JC, Grewal R (2012) Effectiveness of different methods of resistance exercises in lateral epicondylosis-a systematic review. J Hand Ther 25: 5-25.

34. Peterson M, Butler S, Eriksson M, Svardsudd K (2014) A randomized controlled trial of eccentric vs. concentric graded exercise in chronic tennis elbow (lateral elbow tendinopathy). Clin Rehabil 28: 862-872.

35. Malliaras P, Barton C, Reeves N, Langberg H (2013) Achilles and patellar tendinopathy loading programmes. a systematic review comparing clinical outcomes and identifying potential mechanisms for effectiveness. Sports Med 43: 267-286.

36. Martinez-Silvestrini JA, Newcomer KL, Gay RE, Schaefer MP, Kortebein P, et al. (2005) Chronic lateral epicondylitis: comparative effectiveness of a home exercise program including stretching alone versus stretching supplemented with eccentric or concentric strengthening. J Hand Ther 18: 411-419.

37. Malliaras P, Cook J, Purdam C, Rio E (2015) Patellar Tendinopathy: Clinical Diagnosis, Load Management, and Advice for Challenging Case Presentations. J Orthop Sports Phys Ther :1-33. 35.

38. Stasinopoulos D (2017a) Strengthening of supinator in the management of Lateral Elbow Tendinopathy. AMJ 10: 373-374.

39. Stasinopoulos D (2017b) Scapular and rotator cuff strengthening in patients with lateral elbow tendinopathy. Hong Kong Physiotherapy Journal 37: 25-26.

40. Juul-Kristensen B, Lund H, Hansen K, Christensen H, DanneskioldSamsøe B, et al. (2008) Poorer elbow proprioception in patients with lateral epicondylitis than in healthy controls: a cross-sectional study. Journal of Shoulder and Elbow Surgery 17(1 Suppl): 72S-81S.

\section{ISSN: 2574-1241}

DOI: 10.26717/BJSTR.2021.39.006329

Dimitrios Stasinopoulos. Biomed J Sci \& Tech Res

This work is licensed under Creative

Commons Attribution 4.0 License

Submission Link: https://biomedres.us/submit-manuscript.php
41. Rio E, Kidgell D, Moseley GL, Gaida J, Docking S, et al. (2015) Tendon neuroplastic training: changing the way we think about tendon rehabilitation: a narrative review. Br J Sports Med 50: 209-215.

42. Alfredson H, Pietila T, Johnson P, Lorentzon R (1998) Heavy load eccentric calf muscle training for the treatment of chronic Achilles tendinosis. Am J Sports Med 26: 360-366.

43. Stanish WD, Rubinovich RM, Curwin S (1986) Eccentric exercise in chronic tendinitis. Clin Orthop Relat Res (208): 65-68.

44. Scattone Silva R, Nakagawa TH, Ferreira AL, Garcia LC, Santos JE, et al. (2016) Lower limb strength and flexibility in athletes with and without patellar tendinopathy. Phys Ther Sport 20: 19-25.

45. Stasinopoulos D (2020) A Questionnaire Survey to Establish Current Clinical Practice of Radial Extracorporeal ShockWave Therapy (rESWT) for the Management of Pain and Functional Impairment on Chronic Lateral Elbow Tendinopathy in Nicosia. J Nov Physiother 10: 443.

46. Karanasios S, Tsamasiotis GK, Michopoulos K, Sakellari V, Gioftsos G (2021) Clinical effectiveness of shockwave therapy in lateral elbow tendinopathy: systematic review and meta-analysis Clin Rehabil.

47. Gaowen Yao, Jing Chen, Yanji Duan, Xiao Chen (2020) Efficacy of extracorporeal shock wave therapy for lateral epicondylitis: a systematic review and meta-analysis. BioMed Res Int: 2064781.

48. Chenxiao Zheng, Dongjie Zeng, Jiayi Chen, Sijing Liu, Jianyi Li, et al. (2020) Effectiveness of extracorporeal shock wave therapy in patients with tennis elbow: a meta-analysis of randomized controlled trials. Medicine 99(30): e21189.

49. Ogden JA, Toth-Kischkat A, Schultheiss R (2001) Principles of shock wave therapy. Clin Orthop Relat Res 387: 8-17.

50. Król P, Franek A, Durmała J, Błaszczak E, Ficek K, et al. (2015) Focused and Radial Shock Wave Therapy in the Treatment of Tennis Elbow: A Pilot Randomised Controlled Study. J Hum Kinet 47: 127-135.

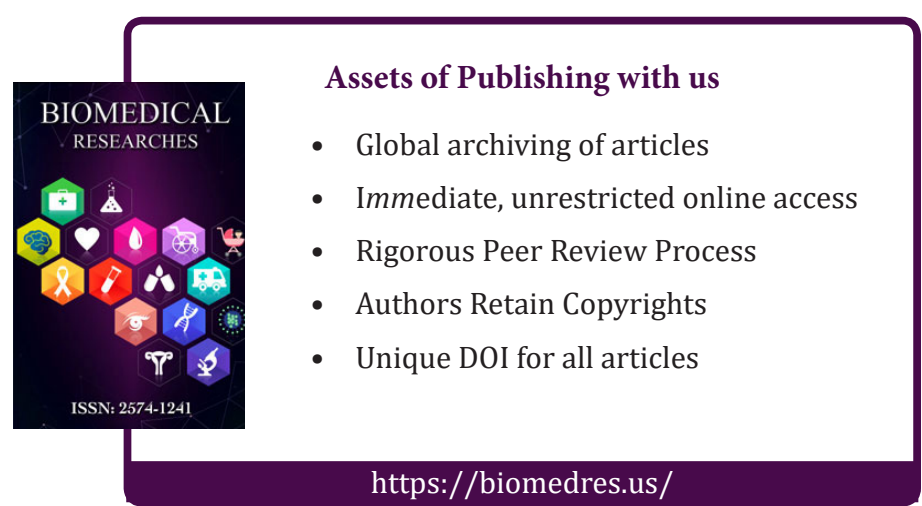

